\title{
Strings with extended non-Abelian gauge interaction
}

\author{
Zdeněk Kopeckÿ* \\ Institute for Theoretical Physics and Astrophysics \\ Masaryk University \\ Kotlářská 2, 61137 Brno \\ Czech Republic
}

\begin{abstract}
The new generalization of the gauge interaction for the bosonic strings is found. We consider some quasiequivariant maps from the space of metrics on the worldsheet to the space of $n$-tuples of one- and two-dimensional loops. The two-dimensional case is based on the cylinders interacted with a path space connection. The special 2-gauge string model is formulated using two 1-connections, non-Abelian background symmetric tensor field and non-Abelian 2-form. The branched non-Abelian space-time is the result of our construction.
\end{abstract}

PACS numbers: 11.25.-w, 04.50.+h

*Electronic address: kopecky@physics.muni.cz 


\section{INTRODUCTION}

Gauge interaction in the open string theory was introduced by adding the Wilson loops of a connection 1-form along each component of the boundary of the string worldsheet. A two-dimensional generalization (2-gauge string theory) of the previous one-dimensional construction is not known. Many motivations come from the formulation of the gauge theory on the path space and especially from the geometry of the non-Abelian Stokes theorem 1, 2 2, 3, 4, 5, 6]. The two-dimensional surface is considered as an ordered one, in other words, covered by a path in the space of paths on the surface. A no-go theorem of Teitelboim 7] states that 2-dimensional non-Abelian gauge interaction is not compatible with the reparametrization invariance.

The works [8, 9, 10, 11, 12, 13, 14, 15, 16, 17] contain some contributions to the 2-gauge string theory building. Their approaches are based on the path space or the gerb geometry.

In this paper the 2-Wilson loops have been incorporated directly into the Polyakov path integral.

\section{STRING WORLDSHEETS WITH MOVING WILSON LOOPS}

Let $\Sigma$ be an oriented string worldsheet modeled by an open or closed orientable compact surface and we denote by $\mathrm{CTop}_{2}^{+}$nonequivalent topologies of such surfaces. Let Met $(\Sigma)$ be the space of Eucledean metrics on the surface $\Sigma$. The string worldsheet is embedded into a target space-time manifold $M$ :

$$
X: \Sigma \rightarrow M
$$

Let the manifold $M$ be the base of a gauge bundle with a compact gauge group and a connection $A$. There is an $n$-tuple of loops $\mathcal{C}=\left(C_{1}, \ldots, C_{n}\right) \in(\mathcal{L} \Sigma)^{n}$ embedded on the surface where $\mathcal{L} \Sigma$ denotes the loop space: $\mathcal{L} \Sigma=C^{\infty}\left(S^{1}, \Sigma\right)$. Our aim is to modify the string partition function by Wilson loops of the connection $A$ around the $n$-tuple $\mathcal{C}$ :

$$
W_{F}^{(1)}[\mathcal{C}]=\int D \gamma D X \exp (-S(\gamma, X)) \prod_{C_{i} \in \mathcal{C}} \operatorname{Tr} P \exp \int_{C_{i}} A
$$

where $S(\gamma, X)$ is the string action in the background $(G, B)$ of metric tensor and antisymmetric form, respectively:

$$
S(\gamma, X)=\frac{1}{2} \int_{\Sigma}\left(G_{\mu \nu} \mathrm{d} X^{\mu} \wedge * \mathrm{~d} X^{\nu}-\sqrt{-1} B_{\mu \nu} \mathrm{d} X^{\mu} \wedge \mathrm{d} X^{\nu}\right) .
$$


Let the positions of the loops in (2) be a general ones not fixed on the boundaries of the worldsheet, as one usually uses in string theory. A dependence of the system loops $\mathcal{C}$ on the surface metric is considered:

$$
\operatorname{Met}(\Sigma) \ni \gamma \rightarrow \mathcal{C}(\gamma)=\left(C_{1}(\gamma), \ldots, C_{n}(\gamma)\right) \in(\mathcal{L} \Sigma)^{n}
$$

The distribution $\mathcal{C}(\gamma)$ of $n$-tuples over the space $\operatorname{Met}(\Sigma)$ in (44) cannot be arbitrary. We impose the quasiequivariant condition on the structure function (44) to save the Weyldiffeomorphism symmetry:

$$
\begin{aligned}
& \forall \gamma \in \operatorname{Met}(\Sigma), \forall w=\left(f, e^{2 \phi}\right) \in \operatorname{Diff}^{+}(\Sigma) \ltimes \operatorname{Weyl}(\Sigma), \\
& \exists \tilde{f_{K}}(\gamma) \in \operatorname{CKD}(\Sigma, \gamma), \exists \tilde{F}^{S^{1}}(\gamma) \in\left(\operatorname{Diff}^{+}\left(S^{1}\right)\right)^{n}, \\
& \exists \tilde{\Pi}(\gamma) \in S_{n}: \mathcal{C}(w(\gamma))=\left(f \tilde{f}_{K}(\gamma)\right)^{-1}\left[\mathcal{C}(\gamma) \circ \tilde{F}^{S^{1}}(\gamma)\right]_{\tilde{\Pi}(\gamma)},
\end{aligned}
$$

where $S_{n} \ni \Pi \mapsto[\ldots]_{\Pi}$ denotes the action of permutation group $S_{n}$ on the loops, $\operatorname{CKD}(\Sigma, \gamma)$ is the group of conformal Killing diffeomorphisms of the metric $\gamma$ and Weyl $(\Sigma)$ is the group of Weyl transformations. The integration over all metrics in (2) substantially depends on the system function $\mathcal{C}$ (44). The property (5) allows us to restrict the integration on the standard moduli space:

$$
\mathcal{M}(\Sigma)=\frac{\operatorname{Met}(\Sigma)}{\operatorname{Diff}^{+}(\Sigma) \ltimes \operatorname{Weyl}(\Sigma)} .
$$

The extended moduli space is introduced

$$
\mathcal{M}_{n}^{C}(\Sigma)=\frac{\operatorname{Met}(\Sigma) \times(\mathcal{L} \Sigma)^{n}}{\sim_{C}}
$$

where $\sim_{C}$ is the relation of equivalence on the space $\operatorname{Met}(\Sigma) \times(\mathcal{L} \Sigma)^{n}$ :

$$
\begin{aligned}
& \sim_{C}:(\gamma, \mathcal{C}) \cong\left(\gamma^{\prime}, \mathcal{C}^{\prime}\right) \Leftrightarrow \gamma^{\prime}=w(\gamma) \text { and } \mathcal{C}^{\prime}=f\left[\mathcal{C} \circ F^{S^{1}}\right]_{\Pi}, \\
& w=\left(f, e^{2 \phi}\right) \in \operatorname{Diff}^{+}(\Sigma) \ltimes \operatorname{Weyl}(\Sigma), F^{S^{1}} \in\left(\operatorname{Diff}^{+}\left(S^{1}\right)\right)^{n}, \Pi \in S_{n} .
\end{aligned}
$$

The extended moduli space (17) can be endowed with the canonical projection to the standard moduli space:

$$
\mathcal{M}_{n}^{C}(\Sigma) \rightarrow \mathcal{M}(\Sigma)
$$


The bundle (91) we call the structure bundle. Let $\Gamma\left(\mathcal{M}_{n}^{C}(\Sigma) \rightarrow \mathcal{M}(\Sigma)\right)$ be the space of sections of the structure bundle (9). Two loop systems $\mathcal{C}$ and $\mathcal{C}^{\prime}$ with the property (515) define a section of the bundle if and if

$$
\forall \gamma \in \operatorname{Met}(\Sigma): \mathcal{C}^{\prime}(\gamma)=\left(\tilde{f}_{K}(\gamma)\right)^{-1}\left[\mathcal{C}(\gamma) \circ \tilde{F}^{S^{1}}(\gamma)\right]_{\tilde{\Pi}(\gamma)},
$$

where $\tilde{F}^{S^{1}}(\gamma) \in \operatorname{Diff}^{+}\left(S^{1}\right), \tilde{\Pi}(\gamma) \in S_{n}$ and $\tilde{f}_{K}(\gamma) \in \operatorname{CKD}(\Sigma, \gamma)$.

The resulting path integral will depend on a sequence of extended bundle sections sets. The sequence is parametrized by the topology discrete parameter $g \in \mathrm{CTop}_{2}^{+}$:

$$
\mathcal{S}_{g}=\left\{s_{m} \in \Gamma\left(\mathcal{M}_{n_{m}}^{C}\left(\Sigma_{g}\right) \rightarrow \mathcal{M}\left(\Sigma_{g}\right)\right), m \in \mathbf{N}, n_{m} \in \mathbf{N}_{\mathbf{0}}\right\}
$$

Now we are able to write explicitly the partition function

$$
W_{F}^{(1)}\left[\left\{\mathcal{S}_{g}\right\}\right]=\sum_{g} \sum_{s \in \mathcal{S}_{g}} \int_{\mathcal{M}_{g}}[D \gamma] \int D X \exp (-S(\gamma, X)) \prod_{C_{i}(\gamma) \in \mathcal{C}(\gamma)} \operatorname{Tr} P \exp \int_{C_{i}(\gamma)} A,
$$

where $\mathcal{C}(\gamma)$ is a representative for the element $s \in \mathcal{S}_{g}$ and $[D \gamma]$ is the integral over the moduli space $\mathcal{M}_{g}=\mathcal{M}\left(\Sigma_{g}\right)$.

The sum over closed surfaces with trivial non-intersected loops provides an example of Eq. (12) for $n_{m}=m$.

\section{STRING WORLDSHEETS WITH 2-GAUGE INTERACTION}

In this section we apply the method of moving loops to the case of two-dimensional cylinders on the worldsheet.

A proposal for 2-gauge string model in the context of the path group approach was given in [8]. Further applications of differential geometry on the path space are contained in [5, 6].

\section{A. String interaction with path space connection}

We denote by $\mathcal{P} Y$ the space of smooth paths on a manifold $Y$. The space of smooth loops on $Y$ will be denoted by $\mathcal{L} Y$ and $\mathcal{L P} Y=C^{\infty}\left(S^{1} \times[0,1], Y\right)$ is the space of cylinders on $Y$. Let a principal $G$-bundle $P_{G}=P(\mathcal{P} M, G)$ be built on the space $\mathcal{P} M$ with the compact structure gauge group $G$ and the bundle geometry is governed by a $G$-connection $\mathcal{A}$. An 
embedding $X: \Sigma \rightarrow M$ from the string worldsheet to the target space $M$ induces the following map in the path spaces:

$$
\widetilde{X}: \mathcal{P} \Sigma \rightarrow \mathcal{P} M, \mathcal{P} \Sigma \ni p \rightarrow X \circ p
$$

We pull back the $G$-bundle $P_{G}$ and consequently the connection $\mathcal{A}$ using the map $\widetilde{X}$ on the space $\mathcal{P} \Sigma$. The holonomy of the connection $\mathcal{A}$ along a loop in the space $\mathcal{P} \Sigma$ represented by a cylinder $\mathrm{Cyl} \in \mathcal{L} \mathcal{P} \Sigma$

$$
P \exp \int_{\mathrm{Cyl}} \mathcal{A}
$$

will be the basic geometry object in our construction. The path-ordered exponential in Eq. (14) is taken with respect to the $S^{1}$ argument of the cylinder Cyl. Let $n$-tuples of cylinders be distributed over $\operatorname{Met}(\Sigma)$ :

$$
\operatorname{Met}(\Sigma) \ni \gamma \rightarrow \mathcal{C}^{2}(\gamma)=\left(\operatorname{Cyl}_{1}(\gamma), \ldots, \operatorname{Cyl}_{n}(\gamma)\right) \in(\mathcal{L P} \Sigma)^{n}
$$

The string path integral will be modified in a similar way as in the one-dimensional case by the product of traces of holonomies connected with the $n$-tuple:

$$
W^{(2)}\left[\mathcal{C}^{2}(\gamma)\right]=\prod_{i=1}^{n} \operatorname{Tr} P \exp \int_{\operatorname{Cyl}_{i}(\gamma)} \mathcal{A}
$$

The distribution (15) cannot be arbitrary to reduce the $\gamma$-integration in the path integral to the moduli space (6), therefore, we restrict (15) by the quasiequivariant condition

$$
\begin{aligned}
& \forall \gamma \in \operatorname{Met}(\Sigma), \forall w=\left(f, e^{2 \phi}\right) \in \operatorname{Diff}^{+}(\Sigma) \ltimes \operatorname{Weyl}(\Sigma), \\
& \exists \tilde{f_{K}}(\gamma) \in \operatorname{CKD}(\Sigma, \gamma), \exists \tilde{F}(\gamma) \in(\operatorname{Diff}[\operatorname{Cyl}])^{n}, \\
& \exists \tilde{\Pi}(\gamma) \in S_{n}: \mathcal{C}^{2}(w(\gamma))=\left(f \tilde{f}_{K}(\gamma)\right)^{-1}\left[\mathcal{C}^{2}(\gamma) \circ \tilde{F}(\gamma)\right]_{\tilde{\Pi}(\gamma)},
\end{aligned}
$$

where Diff $[\mathrm{Cyl}]$ is given for a general connection:

$$
\operatorname{Diff}[\mathrm{Cyl}]=\operatorname{Diff}^{+}\left(S^{1}\right) \times I d,
$$

and in the particular case of reparametrization invariant connection:

$$
\operatorname{Diff}[\mathrm{Cyl}]=\operatorname{Diff}^{+}\left(S^{1}\right) \times \operatorname{Diff}^{+}([0,1])
$$


The extended moduli space which corresponds to the modification of the string path integral by the term (16) is

$$
\mathcal{M}_{n}^{C^{2}}(\Sigma)=\frac{\operatorname{Met}(\Sigma) \times(\mathcal{L P} \Sigma)^{n}}{\sim_{C^{2}}}
$$

where the equivalence relation is given by

$$
\begin{aligned}
& \sim_{C^{2}}:\left(\gamma, \mathcal{C}^{2}\right) \cong\left(\gamma^{\prime}, \mathcal{C}^{2}\right) \Leftrightarrow \gamma^{\prime}=w(\gamma) \text { and } \mathcal{C}^{2}=f\left[\mathcal{C}^{2} \circ F\right]_{\Pi}, \\
& w=\left(f, e^{2 \phi}\right) \in \operatorname{Diff}^{+}(\Sigma) \ltimes \operatorname{Weyl}(\Sigma), F \in(\operatorname{Diff}[\mathrm{Cyl}])^{n}, \Pi \in S_{n} .
\end{aligned}
$$

The structure bundle of the extended moduli space (20) is

$$
\mathcal{M}_{n}^{C^{2}}(\Sigma) \rightarrow \mathcal{M}(\Sigma)
$$

Finally, the partition function for our strings with the cylinder Wilson loops reads

$$
W_{F}^{(2)}\left[\left\{\mathcal{S}_{g}\right\}\right]=\sum_{g} \sum_{s \in \mathcal{S}_{g}} \int_{\mathcal{M}_{g}}[D \gamma] \int D X \exp (-S(\gamma, X)) \prod_{\mathrm{Cyl}_{i}(\gamma) \in \mathcal{C}^{(2)}(\gamma)} \operatorname{Tr} P \exp \int_{\mathrm{Cyl}_{i}(\gamma)} \mathcal{A}
$$

where

$$
g \in \mathrm{CTop}_{2}^{+}, \mathcal{S}_{g}=\left\{s_{m} \in \Gamma\left(\mathcal{M}_{n_{m}}^{C^{2}}\left(\Sigma_{g}\right) \rightarrow \mathcal{M}\left(\Sigma_{g}\right)\right), m \in \mathbf{N}, n_{m} \in \mathbf{N}_{\mathbf{0}}\right\}
$$

and $\mathcal{C}^{2}(\gamma)$ is a representative for the element $s \in \mathcal{S}_{g}$.

\section{B. Special 2-gauge strings}

In this part a non-Abelian string interaction is formulated using geometrical objects connected with the bundle of horizontal paths [6]. The horizontal paths with respect to a connection $A$ on a given principal bundle $P$ create the principal $G$-bundle $\mathcal{P}_{A}$ that is an example of principal $G$-bundle on the path space. A connection $\bar{A}$ and a non-Abelian 2 -form $B$ define the special connection on the $G$-bundle $\mathcal{P}_{A}[6]$.

Let $R$ be an irreducible representation of the compact simple gauge group $G$. The product

of $R$ and its contragradient representation $\bar{R}$ can be expanded to the direct sum of irreducible representations $R_{i}$ :

$$
R \otimes \bar{R}=\sum_{i} R_{i}
$$


The group $G$ acts by the similarity transformation on the space of the complex $N \times N$ matrices $M_{N}(C)$ where $N=\operatorname{dim}_{C} R$. The invariant subspace of the Hermitian matrices

$$
\operatorname{Herm}(N) \subset M_{N}(C)
$$

can be written as the real sum of the irreducible subspaces

$$
\operatorname{Herm}(N)=\sum_{i} V_{R_{i}}
$$

where $V_{R_{i}}$ is the representation space for the representation $R_{i}$ from the expansion (25). Let $P_{C P}$ be a fixed principal $G$-bundle over $M$, and we generate from the bundle $P_{C P}$ the associate vector bundles

$$
E_{V_{R_{i}}}=P_{C P} \times_{R_{i}} V_{R_{i}}
$$

Our aim is to generalize the action (3) by changing the symmetric tensor $G_{\mu \nu}$ and the antisymmetric tensor $B_{\mu \nu}$ by the bundle (28) valued objects, in other words, they will be sections of the bundles $T_{S y m}^{2}\left(M, E_{V_{R i}}\right)$ and $\Omega^{2}\left(M, E_{V_{R^{\prime}}}\right)$, respectively.

We begin with putting together an embedded cylinder $\mathrm{Cyl} \in \mathcal{L} P \Sigma$, bundle (28) valued tensors $(G, B)$, and a pair of connections $(A, \bar{A})$ in the representation $R$. As an extension of the holonomy construction for the special connection [6], a gauge invariant quantity, which comprises all these objects, can be constructed:

$$
W^{(2)}[\mathrm{Cyl}]=\operatorname{Tr} P_{s} \exp \left[-\frac{1}{2} \int_{0}^{1} d s \operatorname{Hol}(\bar{A}, s)^{-1} S H(G, B, A, s) \operatorname{Hol}(\bar{A}, s)\right],
$$

where

$$
\begin{aligned}
& \operatorname{Hol}(\bar{A}, s)=P_{\bullet} \exp \int_{\mathrm{Cyl}(\bar{\bullet}, 0)} \bar{A}, \bar{\bullet}=[0, s], \\
& S H(G, B, A, s)= \\
& \int_{0}^{1} d t\left[u_{A}(s, t)^{-1} \mathrm{Cyl}_{(s, t)}^{*}\left(G_{\mu \nu} \mathrm{d} X^{\mu} \wedge * \mathrm{~d} X^{\nu}-\sqrt{-1} B_{\mu \nu} \mathrm{d} X^{\mu} \wedge \mathrm{d} X^{\nu}\right) u_{A}(s, t)\right], \\
& \mathrm{Cyl}_{(s, t)}^{*}(\omega)=\mathrm{Cyl}^{*}(\omega)\left(\frac{\partial}{\partial s}, \frac{\partial}{\partial t}\right)(s, t), \omega \in \Omega^{2}(\Sigma), \\
& u_{A}(s, t)=P \bullet \exp \int_{\mathrm{Cyl}(s, \bullet)} A, \bullet=[0, t],(s, t) \in S^{1} \times[0,1] .
\end{aligned}
$$


Eq. (29) is invariant with respect to the local gauge transformation generated by a function $\tilde{g}(x) \in G$ on $M$ :

$$
\begin{aligned}
A & \rightarrow \tilde{g} A \tilde{g}^{-1}+d \tilde{g} \tilde{g}^{-1}, \\
\bar{A} & \rightarrow \tilde{g} \bar{A} \tilde{g}^{-1}+d \tilde{g} \tilde{g}^{-1}, \\
B & \rightarrow \tilde{g} B \tilde{g}^{-1}, \\
G & \rightarrow \tilde{g} G \tilde{g}^{-1} .
\end{aligned}
$$

The disc is the result of transforming the $\bar{A}$-boundary of a cylinder

$$
\operatorname{Cyl}(\bar{\bullet}, 0), \boldsymbol{\bullet}=[0,1]
$$

into a point. In the disc case Eq. (29) is simplified to

$$
W^{(2)}[\mathrm{Disc}]=\operatorname{Tr} P_{s} \exp \left[-\frac{1}{2} \int_{0}^{1} d s S H(G, B, A, s)\right] .
$$

The partition function can be directly formulated:

$$
W_{F}^{(2)}\left[\left\{\mathcal{S}_{g}\right\}\right]=\sum_{g} \sum_{s \in \mathcal{S}_{g}} \int_{\mathcal{M}_{g}}[D \gamma] \int D X \prod_{\operatorname{Cyl}_{i}(\gamma) \in \mathcal{C}^{2}(\gamma)} W^{(2)}\left[\operatorname{Cyl}_{i}(\gamma)\right],
$$

where $W^{(2)}$ and $\mathcal{S}_{g}$ are given by (29) and (24), respectively. In a general model, the pair $(G, B)$ for each cylinder in (40) can be taken as the sum of irreducible parts with coupling constants $\lambda_{i}^{(r)}$ and $\tilde{\lambda}_{i}^{(r)}$ :

$$
G_{i}=\sum_{r} \lambda_{i}^{(r)} G^{(r)}, B_{i}=\sum_{r} \tilde{\lambda}_{i}^{(r)} B^{(r)},
$$

where $i$ and $r$ are indices for the cylinders and bundles, respectively. The extended moduli space (20) with (19) is valid for the model (40) but we do not permute the cylinders in (17) with the different gauge doublets (41). We call the model (40) special 2-gauge strings.

Let us discuss important configurations within the construction of 2-gauge strings:

\section{- Closed or open surfaces with one or two discs}

As an example of Eq. (40), we add one or two discs on a surface $\Sigma$ :

$$
\begin{aligned}
& I: \quad\left(\Sigma, \operatorname{Disc}_{1}^{(+)}, \operatorname{Disc}_{2}\right), \\
& I I:\left(\Sigma, \operatorname{Disc}_{1}^{(+)}\right) .
\end{aligned}
$$


The discs in (42) and (43) cover the whole surface $\Sigma$. The structure function $\mathcal{S}_{g}$ is given simply by $\left[\bar{\gamma},\left(\operatorname{Disc}_{1}^{(+)}\left\{, \operatorname{Disc}_{2}\right\}\right)\right]$ for each $g \in \mathrm{CTop}_{2}^{+}$and $\bar{\gamma}$ is a section of the bundle $\operatorname{Met}(\Sigma) \rightarrow \mathcal{M}(\Sigma)$. The sign (+) in (42) and (43) means the same orientation compared with the orientation of the surface. The partition function (40) for the configurations (42) and (43) reads

$$
\begin{aligned}
I: & W_{F}^{(2)}\left[\left\{\mathcal{S}_{g}\right\}\right]=\sum_{g} \int_{\mathcal{M}_{g}}[D \gamma] \int D X W^{(2)}\left[\operatorname{Disc}_{1}^{(+)}\right]_{S} W^{(2)}\left[\operatorname{Disc}_{2}\right]_{N A}, \\
I I: & W_{F}^{(2)}\left[\left\{\mathcal{S}_{g}\right\}\right]=\sum_{g} \int_{\mathcal{M}_{g}}[D \gamma] \int D X W^{(2)}\left[\operatorname{Disc}_{1}^{(+)}\right]_{S+N A},
\end{aligned}
$$

here $S$ denotes the pair $(G, B)$ in the singlet representation, i.e., proportional to the unit matrix and $N A$ is the non-Abelian part.

\section{- Open stringlike configuration}

Let an open string surface $\Sigma$ be composed in the form

$$
\left(\Sigma, \operatorname{Disc}^{(+)}, \mathrm{Cyl}_{1}, \ldots, \mathrm{Cyl}_{n-1}\right),\left(\operatorname{Disc}^{(+)}, \mathrm{Cyl}_{1}, \ldots, \mathrm{Cyl}_{n-1}\right) \in(\mathcal{L} \mathcal{P}(\Sigma))^{n}
$$

where $n-1$ is the number of holes. In (46) the disc and $\bar{A}$-boundaries (38) of the cylinders cover the whole surface and its boundaries, respectively. Let $\bar{\gamma}$ be a section of the bundle $\operatorname{Met}(\Sigma) \rightarrow \mathcal{M}(\Sigma)$. The structure function is taken $\left[\bar{\gamma},\left(\operatorname{Disc}^{(+)}, \mathrm{Cyl}_{1}, \ldots, \mathrm{Cyl}_{n-1}\right)\right]$. The partition function (40) for the open configuration is

$$
W_{F}^{(2)}\left[\left\{\mathcal{S}_{g}\right\}\right]=\sum_{g} \int_{\mathcal{M}_{g}}[D \gamma] \int D X W^{(2)}\left[\operatorname{Disc}^{(+)}\right]_{S} \prod_{i=1}^{n-1} W^{(2)}\left[\mathrm{Cyl}_{i}\right]_{N A},
$$

where the cylinders induce non-Abelian interaction.

The insertion of the $\bar{A}$-holonomy (30) in Eq. (29) yields

$$
W^{(2)}[\mathrm{Cyl}]=\operatorname{Tr}\left\{\operatorname{Hol}(\bar{A}, 1) P_{s} \exp \left[-\frac{1}{2} \int_{0}^{1} d s \operatorname{Hol}(\bar{A}, s)^{-1} S H(G, B, A, s) \operatorname{Hol}(\bar{A}, s)\right]\right\} \cdot
$$

Using (48) in Eq. (47) we get the open string partition function for the configuration when the cylinders fields $(G, B)$ are zero. A model with moving Wilson loops is recovered if we exchange for the cylinder the $\bar{A}$-boundary (38) and the free boundary

$$
\operatorname{Cyl}(\bar{\bullet}, 1), \bar{\bullet}=[0,1] .
$$




\section{- Non-Abelian branched space-time}

The singlet part in (44) and (45) stabilizes space-time and the non-Abelian part causes a branching of space-time. The Abelian and non-Abelian terms are separated by the new coupling constants.

\section{CONCLUSIONS}

In this paper, the new gauge interaction has been obtained for bosonic string. The path integral is equipped by moving one- and two-dimensional gauge loops. The resulting partition function has acquired as a parameter the large moduli space dependence.

Straightforwardly, it is possible to generalize the special 2-gauge strings construction (40) by adding non-Abelian dilaton or tachyon terms.

As a consequence of the space-time non-Abelian structure, some nontraditional Higgs mechanism can be expected. The model (45) corresponds to an interacted parallel spacetime. Such bigravity interaction in the field limit has been studied in the literature [18] with cosmological applications.

The special connection on the space of horizontal path can be iterated into a higher connection on the multiple horizontal path space [6]. The higher connection is generated by a tower of non-Abelian $p$-forms $(p \geq 1)$ and its Wilson loop can describe the non-Abelian interaction of a higher dimensional brane.

\section{Acknowledgments}

It is a pleasure to thank J. Horský for fruitful conversation and useful comments on the manuscript.

[1] A. M. Polyakov, Nucl. Phys. B 164, 171 (1980).

[2] P. G. O. Freund and R. I. Nepomechie, Nucl. Phys. B 199, 482 (1982).

[3] R. I. Nepomechie, Nucl. Phys. B 212, 301 (1983). 
[4] M. B. Mensky, Path Group: Measurements, Fields, Particles (Nauka, Moscow, 1983), in Russian.

[5] O. Alvarez, L. A. Ferreira, and J. S. Guillen, Nucl. Phys. B 529, 689 (1998).

[6] A. S. Cattaneo, P. Cotta-Ramusino, and M. Rinaldi, Commun. Math. Phys. 204, 493 (1999).

[7] C. Teitelboim, Phys. Lett. B 167, 63 (1986).

[8] M. B. Mensky, Sov. Phys. JETP 63, 239 (1986).

[9] A. Kapustin, Adv. Theor. Math. Phys. 4, 127 (2000).

[10] J. Kalkkinen, hep-th/9910048.

[11] I. Chepelev, J. High Energy Phys. 02 (2002) 13.

[12] C. Hofman, hep-th/0207017.

[13] H. Pfeiffer, Ann. Phys. (N.Y.) 308, 447 (2003).

[14] G. Florian and H. Pfeiffer, J. Math. Phys. (N.Y.) 45, 3949 (2004).

[15] A. Lahiri, Int. J. Geom. Meth. Mod. Phys. 1, 299 (2004).

[16] U. Schreiber, hep-th/0407122.

[17] P. Aschieri and B. Jurčo, J. High Energy Phys. 10 (2004) 68.

[18] T. Damour, I. I. Kogan, and A. Papazoglou, Phys. Rev. D 66, 104025 (2002). 